\title{
Enhancing Accessibility for University Students with Specific Learning Disabilities Using a Computerized Screening System
}

\author{
Marcus Henning*
}

\author{
Centre for Medical and Health Sciences Education, The University of Auckland, Private Bag 92019, Auckland 1142, \\ New Zealand
}

\begin{abstract}
This paper considers the diagnostic value of a computer screening system as an alternative, or adjunct, to traditional battery testing systems in identifying the presence of specific learning disabilities (SLD) in university students. In the present times of increasing numbers of students with various forms of disabilities entering tertiary education, institutional fiscal constraints, problems with employing and securing professional staff, and the heightened value placed on time, it would seem sensible to utilize cost and time efficient systems for diagnosing the presence of disabilities in students. This paper therefore considers the use of the Lucid Adult Dyslexia Screening (LADS) system, and draws from the university casebook of one psychologist to highlight its usefulness in terms of increasing accessibility to assessment and associated services. It is argued that, because the diagnostic phase is crucial to the approval and implementation of accommodations, efficiency in the assessment process contributes to reducing barriers that students with SLD may face in gaining access to equitable assistive resources. The conclusion suggests that LADS is a cost and time efficient system, but needs to be used judiciously by considering the clinical history of the student. Scope for further research is also indicated.
\end{abstract}

Keywords: Learning disability, adult dyslexia, diagnostic software, university students with disabilities.

\section{INTRODUCTION}

The assessment and didactic implications of specific learning disabilities (SLD) are critical discussion points that need to be continually addressed by higher educationalists [1]. The use of diagnostic systems for determining the presence or absence of a SLD is necessary, as it creates a platform for implementing effective interventions to address the problems facing students with this type of disability. This paper considers the issues of diagnosis and intervention within the university system. It further presents a case example that draws from the clinical and educational experiences of the present author who initiated the use of a computerized screening system to improve accessibility to timely assessment for students with SLD and to generate more opportunities for assistance from disability agencies and faculty within the university.

A general definition of SLD was provided at the Center on Disabilities Technology and Persons with Disabilities Conference in 1997 at the California State University [2]. It states that SLD is

a disorder in one or more of the central nervous system processes involved in perceiving, understanding and/or using concepts through verbal (spoken or written) language or nonverbal means. This disorder manifests itself with a deficit in one or more of the following areas: attention, reasoning, processing, memory, communication, reading, writing, spelling, calculation, coordination, social competence and emotional maturity.

*Address correspondence to this author at the Centre for Medical and Health Sciences Education, The University of Auckland, Private Bag 92019, Auckland 1142, New Zealand; Tel: 0064-9-3737599, Ext. 87392; 0064-93737204; E-mail: m.henning@auckland.ac.nz
It is important to note however that definitions of SLD have developed over recent times. Jordan [3] suggests that definitions of SLD went through several iterations such that by the early 1980 s there were more than 200 definitions of learning disability that were publicly available, and further refinements continued into the 1990s. Jordan further states that in the 1990s several common concepts were being developed; specifically these were: learning disability, learning difference, learning difficulty, and late development. A further term used interchangeably with SLD is dyslexia. Dyslexia is defined as a disorder of single word reading or spelling, and there is often a problem with working memory and left-right discrimination [4]. Differences between the two terms are difficult to discern and depend on the usage of test batteries, different legal systems, tradition and professional inclination in terms of using diagnostic labels, and regional and geographical preferences. In the UK, the term dyslexia appears to be more commonly used whereas in the US the use of SLD is more prevalent [5]. Nonetheless, SLD is a more generic term and includes dyslexia which is a more specific term suggesting the presence of a reading disorder $[5,6]$.

Currently, the ability-achievement discrepancy determination method is the most widespread system for determining the presence of SLD [7]. The IQ-achievement discrepancy determines the presence of SLD if students score below their IQ expectations in specific areas, such as reading, writing and mathematics [8]. There is evidence that this diagnostic system provides a platform for the implementation of suitable interventions, in the form of accommodations which increase the probability of academic attainment for students with SLD within educational settings [9]. However, Kavale [10] contends that the predominant definition of SLD, based on an exclusive discrepancy notion, 
has problems with it being too 'vague' in that it has a wide brief, but in its expansiveness is also unlikely to be wrong: thus students may be diagnosed with SLD even if this may not be the case. Additional concerns with the use of the discrepancy method include consistency across tests, temporal stability of results, validity problems in terms of decision making, and the implementation of effective and timely interventions [7]. The problems associated with definition also affect the accuracy of determining the prevalence of SLD [11].

The worldwide prevalence of students with SLD in higher education may be as high as $10-12 \%$ [12]. Other more conservative estimates of prevalence range from $2-10 \%[6$, $8,13]$. In addition, it is four times more likely for males to have SLD [14]. However, some recent research suggests that this gender difference may not be so marked [13]. The problems in determining exact prevalence are further complicated by different combinations of SLD, such that " $2.3 \%$ had combined reading and arithmetic disabilities, $3.9 \%$ had a disability in reading only, and $1.3 \%$ had a disability in arithmetic only" [13].

The problems associated with the discrepancy theory have generated an interest in refining the determination of SLD. Kavale [10] has put forward a responsiveness to intervention (RTI) theory in relation to determining the presence or absence of SLD. The RTI theory of determination relies on two steps, specifically identification of a learning problem and implementation of an appropriate intervention strategy. In contrast, the discrepancy theory focuses purely on identification of a specific disorder in relation to an overall IQ measure [8]. The RTI system is an outcome-based model, whereby students are identified as having problems in a specific area, such as reading, and are then provided with an authenticated intervention. If students are able to markedly improve in their areas of learning deficit, then a diagnosis of SLD would not be made. However, if students are unable to respond then a diagnosis of SLD would be warranted. As such, the RTI system has a "non-responsiveness" basis to its decision making formula. The rationale that underpins this formula requires greater clarification, given that some interventions are designed to address the learner's SLD and thus would be expected to be effective despite the presence of the SLD.

The process to ascertain the presence or absence of SLD among students in New Zealand universities is fairly standardized and is based primarily on using a discrepancy approach [15]. The process for determining the presence of SLD requires authentication from several sources. These include: (i) personal history; (ii) clinical presentation in terms of educational and medical history; (iii) letters or reports from specialists in areas such as educational psychology, neuropsychology, neurology or psychiatry; and (iv) verifiable findings from an appraisal using a battery of psychological tests. This information provides the basis for a decision that culminates in whether or not students are eligible for assistance in line with the provisions allocated to students with SLD [16].

In the US, in diagnosing SLD, the psychometric tests most often employed are the Wechsler tests, the Wide Range Achievement Test-Revised, Woodcock-Johnson PsychoEducational Battery-Revised, and the Bender Visual Motor
Gestalt Test [8]. In the United Kingdom, tests such as the Bangor Dyslexia Test, the Dyslexia Screening Test, the Cognitive Profiling System, and the Lucid Adult Dyslexia Screening (LADS) program are used for screening adult dyslexia [17, 18]. In Australasia, similar test batteries as in the US and the United Kingdom are used. However, the use of these tests presents problems in terms of cultural applicability $[15,19]$. Consequently, it is reasonable to assert that there are differences in diagnostic systems based on linguistic, legal, political, educational, medical, cultural and geographical preferences.

Putting these seemingly insurmountable differences and difficulties aside and considering them as potential confounding variables, the present author decided to investigate the efficacy of a computerized diagnostic system with the aim of determining whether this system could assist in appropriately and efficiently identifying and assisting students with SLD. Given that in the institution concerned all the students who sought help in 2009 did so because of a reading and writing difficulty, the system considered most appropriate was the LADS [20, 21].

\section{THE LUCID ADULT DYSLEXIA SCREENING (LADS) PROGRAM}

LADS is a computerized system that tests certain areas of cognitive skills, namely word recognition, word construction, working memory, and reasoning ability [20]. Word recognition requires the respondent to utilize lexical decoding to discern real words from non-real words. Word construction requires respondents to produce a coded nonreal word from a set of three syllables. Working memory utilizes a backwards digit span approach whereby the respondent hears a set of digits and has to key in the numbers backwards. Finally, for the reasoning ability task, the respondent views a set of patterns and has to determine the pattern that makes sense in completing a specified sequence.

The word recognition, word construction, working memory test are considered difficult for people with dyslexia $[4,20]$. The reasoning task becomes an anchor and provides a measure of general intelligence. The reasoning task provides a frame of reference so that detection of dyslexia can be made based on the discrepancy approach.

In LADS, easier items are delivered first and each test has a time component. If a student has profound difficulties with word recognition, then a long horizontal red bar appears on the report. If the difficulties are evident but not profound, a horizontal orange bar is shown; and if no difficulties are revealed, a green bar appears. The range of the bar is from 1 to 9 on each subtest: the higher the score the greater the weakness in the area being measured [4]. The colour bar for the reasoning ability task is blue and is vertical with five segments.

Lucid Research Ltd [20] emphasizes that LADS is not a diagnostic test per se, but a useful mechanism for determining the likelihood of dyslexia. Thus, a full diagnosis is recommended especially in ambiguous cases. Singleton et al. [21] suggest that the use of a LADS system is ideal in tertiary institutions due to the large student populations. LADS is designed for the 16 years and older age group, and is thus suitable for the university student cohort. It is also comparatively "more affordable" as students can receive 
immediate feedback on the probability of dyslexia without incurring a high psychologist consultation and assessment fee. It is accessible for teachers, and the resulting information can be incorporated in efforts to improve teaching and learning strategies.

The areas of word recognition, word construction, working memory and reasoning were chosen as they are areas of cognition that are indicated in classical definitions of dyslexia [20], namely phonological processing, lexical access, working memory, and speed of information processing. These areas are more difficult to compensate for and are likely to persist throughout the affected students' lives; they are also less likely to be influenced by environmental factors.

Some studies have evaluated the LADS. First, Simmons and Singleton [4] found no significant difference between dyslexic and non-dyslexic groups on the working memory task. However, their sample size of 19 per group was low and this may have hampered the test of significance, given that mean differences were noted (dyslexia group yielded a higher mean score). A more recent study [21] investigated the usefulness of computerized screening for dyslexia in adults. The Wide Range Achievement Test (WRAT-3) and in particular the Reading and Spelling tests from WRAT-3 were incorporated as frames of reference [22]. Singleton et al. found that the LADS obtained $90 \%$ sensitivity and specificity rates for screening dyslexia (excluding the 'borderline' category). In their study, the "borderline category accounted for 21 participants (16\% of the sample) and comprised one-third dyslexics and two-thirds nondyslexics" [21]. The results suggest that there is scope for research into more refined sub-groupings involving individuals who have difficulties with working memory but not with word recognition and word construction.

A further study investigated the differences in a Tutor Screening Battery (TSB) and the LADS with respect to the diagnosis of dyslexia, dyspraxia and Meares-Irlen syndrome [23]. The TSB approach utilized parts of various well-known British dyslexia tests, specifically the newer and older versions of the Bangor Dyslexia Test [24] and the Dyslexia Adult Screening Test (DAST) [25]. The results of this study showed that the TSB system had a more robust overall performance. However, the LADS had higher specificity, but lower sensitivity. According to the authors [23], the LADS will likely miss a higher proportion of students with SLD. In contrast, the TSB system resulted in more false alarm cases.

Moreover, Singleton et al. [21] state that the DAST has 11 subtests that measure phonological processing and auditory memory. Furthermore, the DAST takes about 30 minutes to administer and it may have problems with validation and standardization. Moreover, the sensitivity and specificity rates for DAST were markedly lower than those obtained for the LADS [21]. These findings and observations have implications with respect to cost, time, and minimising student stress levels [23]. It was for these reasons that the present author trialled the use of the LADS on a student population presenting with SLD in New Zealand. The following statistics were taken from the casebook of the present author who practised at a New Zealand-based university as a registered psychologist.

\section{PSYCHOLOGIST-STUDENT CONSULTATION STA- TISTICS}

The operational definition of SLD/dyslexia that was used here was based on the ability-achievement discrepancy determination method [6]. Moreover, follow-up appointments and systematic monitoring systems with faculty and students were employed to ensure that the academic and examination accommodations were consistent with the diagnostic considerations.

The resulting data were considered useful in determining the decision to move from a traditional test-battery system to a computer screening approach (followed by a test-battery system if ambiguities in the LADS diagnosis were indicated). The purpose of Table $\mathbf{1}$ is to illustrate that there was a clear trend of increasing numbers of students with learning disability issues visiting the psychologist for assessment. This trend suggested that something needed to be done to cater for the needs of these students with respect to ensuring the provision of equitable academic and examination accommodations. Moreover, the financial and staffing constraints of the university meant that there was a need for a more efficient system for diagnosis. Hence, the implementation of the LADS was a practical and exploratory strategy to deal with the increasing numbers of students.

Table 1 presents some general information regarding the students seen in connection with SLD for the period 2002 to 2008. Total enrolments for this university in 2008 were 23,715 students (counting both full-time and part-time students), or 16,822 full-time equivalent students; an increase of $2.7 \%$ on the previous year [26]. The frequency count data show several trends. First, the number of actual learning assessments increased markedly over the period of 2002 to 2008 from 40 to 94 . Second, the number of memos sent to faculty staff concerning students presenting with a SLD also increased over this period, although 2007 was seen as an unusually high year. Student memos included those written on behalf of students who required learning assessments as well as those who already had past documentation from external assessors. The memos contained accommodation recommendations to the relevant faculty pertaining to the student needs determined from their learning assessment, such as reader/writer, supervision details, computer access, distraction free-room, and extra time.

On average, a traditional learning assessment takes 2-6 hours. The time spent on testing is a minimum of 2-3 hours, then there are 1-2 hours of report writing and finally a debriefing session between student and psychologist to explain the findings and consider options for the future [15]. With the introduction of LADS in 2008, testing time was dramatically reduced, as only 18 students required full follow-up assessments due to an unclear diagnosis in the LADS output or anomalous clinical data. The LADS assessment is dependent upon student response rate but was generally around 30 to 45 minutes in duration (including an immediate debrief and discussion on options for the future). The students, thus, do not have to wait for a one-week turn around while the psychologist writes the report and considers the tests results; LADS provides instantaneous feedback. 
Table 1. Psychologist's Consultation Details: Number of Learning Assessment Reports and Memos for Students with SLD Written During the Period 2002 to 2008

\begin{tabular}{|c|c|c|c|c|c|c|c|}
\hline Type of Support & $\mathbf{2 0 0 8 *}$ & $\mathbf{2 0 0 7}$ & $\mathbf{2 0 0 6}$ & $\mathbf{2 0 0 5}$ & $\mathbf{2 0 0 4}$ & $\mathbf{2 0 0 3}$ & $\mathbf{2 0 0 2}$ \\
\hline \hline Learning assessments & 94 & 90 & 59 & 49 & 58 & 48 & 40 \\
\hline Memos & 110 & 155 & 72 & 83 & 101 & 79 & 74 \\
\hline
\end{tabular}

Using the January to May 2009 consultation statistics for students presenting with SLD, the probability of the presence of SLD can be surmised using the categories (low, borderline, moderate, and high) provided by the LADS system (as presented in Table 2). A borderline (or moderate) categorization was regarded as an apparent sign for the psychologist to examine the presence of SLD in the student further [21]. Such considerations were completed in line with data pertaining to the student's presenting history and their intentions for study. The Table 2 data show that $65 \%$ of the students yielded a high probability diagnosis of dyslexia. The remaining $35 \%$ of students (NA, low, borderline, and moderate) warranted a follow-up (using more traditional methods of assessment and analysis) so that the results could be considered in line with presenting history, as required. The student with NA was unable to initiate one of the tasks, suggesting the need for a more specific follow-up.

\section{ACCOMMODATIONS AND ACADEMIC ASSISTANCE FOR STUDENTS WITH SLD}

The consultation statistics provide details on two facets related to assisting students with SLD, namely diagnosis and intervention. Students are first assessed and then monitored and assisted in their learning.

\section{Diagnostic Considerations}

The statistics in Table $\mathbf{1}$ indicate that 90 learning assessments were completed in 2007 and 94 in 2008. It was assumed that the increased interest in, and need for, learning assessments came from faculty interest in the learning assessment process and this was a further factor in securing the LADS program. This assumption was based on the number of faculty emails and phone calls received by the psychologist in this period, and the fact that the increase was unlikely to have been due to other factors such as increased student enrollments, as the increase in enrollments was far less than the increase in consultation numbers. The Health and Environmental Sciences faculty was the key and most prolific referral agency $(\mathrm{n}=52 ; 58 \%)$, in comparison with the School of Education $(\mathrm{n}=21 ; 23 \%)$ and other faculties ( $\mathrm{n}$ $=17 ; 19 \%$ ). These findings suggest that many students with SLD were, and are, likely engaging in their study without clear knowledge that assistance was/is available.
This finding also highlights different referral rates from different faculties, drawing the conclusion that more students with SLD take courses in Health and Environmental Sciences than in other faculties. This assumption does require more investigation. One possible reason for this anomaly of differential referral rates from different faculties may be linked to disparity in the selection criteria for students at enrollment by faculty. However, the selection criteria for the Health and Environmental Sciences courses are the most stringent of all the faculties in this university, suggesting that the students presenting with SLD are probably only the 'tip of the iceberg'. Accordingly, it was felt that the LADS has the potential to allow for greater accessibility of SLD identification mechanism for students by using a computerized system that would reduce time, cost, and stress and be a more attractive and less invasive option for diagnosis [23].

\section{Intervention Considerations}

In the university system the most common forms of interventions center around accommodation and academic assistance [15]. There are many types of academic and examination assistance available to students with SLD. For example, identification of SLD can engender the provision of reader/writer assistance, a distraction free room, and extra time in assessments. Note-taking assistance during lectures can also be provided. Moreover, advocacy in relation to student negotiation for accommodations with appropriate staff in their faculty or departments can be provided, as well as academic and/or pastoral support [27]. Accommodations provided are in line with those approved by the New Zealand Qualifications Authority suggesting that "alternative assessments need to be considered for diverse students" [28]. Furthermore, it is important to note that there is a legal motivation (as well ethical and educational) behind the implementation of these accommodations. The legal rights of students with disability are embedded in the New Zealand legislative system such as the Human Rights Act 1993 and the New Zealand Bill of Rights Act 1990 that are monitored by the Human Rights Commission [29].

\section{Key Stakeholders}

For the accommodation process to work, several joint ventures need to be considered, namely between students,

Table 2. Probability of an SLD Diagnosis Using LADS for Consultations $(n=23)$ During the Period January to May 2009

\begin{tabular}{|c|c|c|c|c|c|}
\hline \multirow{2}{*}{ SLD Details } & \multicolumn{4}{|c|}{ LADS Diagnostic Categories } \\
\cline { 2 - 6 } & NA & Low & Borderline & Moderate & High \\
\hline \hline Number of students diagnosed under each category & 1 & 7 & 5 & 2 & .09 \\
\hline Probability of dyslexia & .04 & .30 & .22 & .35 \\
\hline
\end{tabular}


faculty staff, and administrators [2]. First, students with SLD need to take some responsibility for their learning as they need to initiate the process of obtaining accommodations. To apply for these procedures, students need to be familiar with the process of obtaining disability assistance. The process is usually initiated by the students either by disclosing they have a disability on their enrollment form or going to a student service office for advice [16]. To execute the second step students need to have some familiarity with the university system, what the educational provider can offer and the rights of students with SLD. Given the problem of retention in the first year [30], students with SLD may be at risk of attrition in this system simply due to not knowing about the details of the system [31]. It is the experience of the present author that systems that can be more easily accessible, such as the LADS, appear to be a more amenable option for students, in comparison to the traditional battery testing approach; thus, the more accessible options contribute to increasing access to accommodations for those students in need of assistance.

Second, faculty need to be encouraged to employ effective and efficient teaching principles to develop inclusion in the teaching environment $[32,33]$. It was felt that the LADS would be more agreeable to faculty staff and this was certainly true of the Health and Environmental Sciences faculty and School of Education. Both the faculty and the school became more confident about referring students because they had heard of the LADS program at the end of 2007, and maintained this confidence in the use of LADS into 2008. Moreover, the information gained from the LADS was used to inform faculty staff (with the student's permission), and students with SLD appeared to improve their learning capabilities as they felt faculty were well advised $[34,35]$.

Third, administrators are required to implement the services to support students with SLD. Administrative staff is directed by specialist personnel usually by receiving a memorandum in the first instance (see Table 1). Verification of the credibility of the student's claim of SLD is sought through authenticated documentation or written confirmation from in-house psychologist/s or other registered psychologists. At universities in New Zealand, these services administer the academic and exam accommodations [16]. These are usually completed in line with the New Zealand Qualification Authority guidelines [36]. The aim of the administration arm of any disability service is to promote the notion of accessibility, and this can be more effectively achieved through the use of systems, such as LADS, which create the scaffold for examination and academic accommodations and promote the availability of assistive technology [37].

\section{CONCLUSIONS}

The aim of this paper was to review the process of assisting students in tertiary education who present with SLD, and to improve on traditional methods of diagnosis that require test batteries. The process begins with identification and continues with intervention. The identification procedures are globally and locally diverse and depend on institutional, geographical and individual differences in terms of assessment preferences. However, the discrepancy theory has been used predominantly as their frame of reference $[7,8]$, although other theories have been developed in more recent times, such as responsiveness to intervention $[10,11]$. Both theoretical domains require the administration of a battery of tests to discern the presence or absence of SLD. These test batteries are often time consuming, stressprovoking and costly to implement and involve the utilization of many tests that can have some negative repercussions for both the psychologist and the student [23]. In a move to simplify the method of identification and to make the process less invasive, computer screening mechanisms have been developed [20]. The consultation statistics shown in this paper indicate increasing numbers of students being assessed and the system chosen to better manage this increased demand was the use of technology. The high likelihood that the LADS will present with a clear indication of the presence of dyslexia is encouraging and its use is deemed as a possible solution to this increased demand. Moreover, the initial feedback and interest from students and faculty suggest that the aim of increased accessibility is, to some extent, being achieved, and that the LADS approach is timely and amenable for both staff and students.

The paper further reflects on the student load for one psychologist. The increased student consultation workload and increased presentation of students with SLD made the traditional systems untenable for one psychologist and, given the fiscal constraints on further expansion in this area, the LADS system appeared a logical consideration. Moreover, to reduce the cost of time and money for the university it seemed sensible to trial a system that would simplify a traditional approach based on the administration of large batteries of tests. In addition, more students could be assisted in defining the underlying characteristics of the learning disability and, hence, by ascertaining with some confidence whether or not they were likely to have dyslexia, a specific area of SLD. It was important for the psychologist at this institution to be aware of the complexity of the issue and that one computer output is what it is - one piece of valuable information, but not the whole story. Other features needed to be taken into consideration with the computer output, namely prior documentation, and the student's clinical and educational history. Additionally, if the output showed a borderline or moderate diagnosis, then further testing or investigation was needed [21, 23]. Even if the computer output presents with a 'high' or 'low' probability of dyslexia, further testing may be warranted depending on the student's past history and their inclination towards further assessment, thus reinforcing a student-centered approach.

The identification process naturally leads to an intervention phase. This phase requires students to be selfadvocates and to apply a sense of self-determination [33]. It also necessitates compliance and assistance from faculty and administrative staff. The use of LADS not only allows students greater accessibility to the knowledge around their learning disability, but also improves their chance of accessing assistance from disability support systems and faculty-based mechanisms [16, 32, 38]. With the increased sense of support, students will likely improve their chances of retention and academic success [1]. The paper postulates that students who have an SLD diagnosis are more likely to be linked into support services and that this will enhance 
accessibility to their studies. This is a reasonable assertion as the evidence that the provision of support alters the success and retention rates of students with SLD does exist in overseas contexts [9].

Nonetheless, there is still debate as to which diagnostic system provides the most effective method for determining the presence or absence of SLD [22], as there are several considerations related to accuracy and efficiency. It is unclear whether the computerised screening system does, or will, enhance accessibility for students with SLD. However, what is clear is that the computerized system does improve efficiency. A major problem with the LADS is that the screening device is not validated for the New Zealand population. Moreover, the existing psychometric data for other populations [23] show the LADS to have some problems with sensitivity as a detector of SLD. This indicates that the LADS could well be missing cases of SLD. This may be a problem given that only the 'ambiguous' cases are required for further testing. As such, the LADS must not be used as a standalone screening test until the issue of both adequate specificity and sensitivity for the target population is resolved. However, this argument can also be applied to existing traditional systems such as the TSB. Consequently, these arguments confirm that further research is of paramount importance. Despite these limitations, this assessment tool (LADS) serves a useful purpose and is a useful adjunct to existing systems. Due to increased student numbers requiring assessment, fiscal constraints and an inability to increase staff numbers, the present author was required to find an alternative system, or adjunct, to the traditional battery based procedures. It is acknowledged that further research is required within the New Zealand context before full confidence in this system can be assured.

Finally, this paper was a review of the process of identification and intervention with respect to students with SLD. As such the discussion utilized a case study approach drawing from the experiences of, and data gathered by, one psychologist within one university. To investigate the generalizability and full usefulness of the LADS, further research is recommended. Some suggested areas include: (i) further psychometric evaluation; (ii) examining the alignment between the output of the LADS and the accommodations provided; (iii) reviewing the cross-cultural suitability of the LADS; (iv) considering the academic attainment implications of accommodations; and (v) undertaking further comparisons of the LADS with traditional approaches.

\section{REFERENCES}

[1] Ruban LM, McCoach DB, McGuire JM, Reis SM. The differential impact of academic self-regulatory methods on academic achievement among university students with and without learning disabilities. J Learn Disabil 2003; 36(3): 270-86.

[2] Wardin M, Daniels C. Definition of specific learning disability. 1997 [cited 2009 October 14]. Available from: http://www.csun. edu/cod/conf/1997/proceedings/120.htm

[3] Jordan DR. Teaching adults with learning disabilities. Malabar, Florida: Krieger Publishing Company 1996.

[4] Simmons R, Singleton C. The mental and written arithmetic abilities of adults with dyslexia. Dyslexia 2006; 12: 96-114.

[5] Ashby V. Dyslexia and specific learning disabilities. 2008 [updated May 9; cited 2009 May 23]. Available from: http://dpi.wi.gov/sped/ ld-dyslexia.html
[6] American Psychiatric Association. The diagnostic and statistical manual of mental disorders (DSM IV). 4th ed. Washington D.C.: American Psychiatric Association 1994.

[7] Reschly DJ, Hosp JL. State SLD identification policies and practices. Learn Disabil Q 2004; 27(4): 197-213.

[8] Brinkerhoff LC, McGuire JM, Shaw SF. Postsecondary education and transition for students with learning disabilities. $2^{\text {nd }}$ ed. Austin: Pro-ed 2002.

[9] Getzel EE. Addressing the persistence and retention of students with disabilities in higher education: Incorporating key strategies and supports on campus. Exceptionality 2009; 16:(4): 207-19.

[10] Kavale KA. Identifying specific learning disability: Is responsiveness to intervention the answer? J Learn Disabil 2005; 38(6): 553-62.

[11] Kavale KA, Spaulding LS, Beam AP. A time to define: Making the specific learning disability definition prescribe specific learning disability. Learn Disabil Q 2009; 32(1): 39-48.

[12] Payne T, Irons E. Learning disabilities resource package: Tools for assessing learning disability and supporting students with learning disability in Australian universities. Hobart: Department of Education Science and Training, The University of Tasmania 2003.

[13] Dirks E, Spyer G, van Lieshout E, Sonneville Ld. Prevalence of combined reading and arithmetic disabilities. J Learn Disabil 2008; 41(5): 460-73.

[14] Hallahan DP, Kauffman JM, Lloyd JW. Introduction to learning disabilities. Boston: Allyn and Bacon 1996.

[15] Henning MA. Cross-cultural usefulness of assessment tools used to identify the presence of a learning disability. In: Manalo E, WongToi G, Eds. Communication skills in university education: The international dimension. Auckland, New Zealand: Pearson, Prentice Hall 2005; pp. 239-51.

[16] Disability Resource Service. Services. n.d. [cited 2009 May 17] Available from: Auckland University of Technology website: http://www.aut.ac.nz/students/studentV̈services/disability /services.htm

[17] Beachama NA, Altyb JL. An investigation into the effects that digital media can have on the learning outcomes of individuals who have dyslexia. Comput Educ 2006; 47(1): 74-93.

[18] Reid G. Dyslexia: A practitioner's handbook. $2^{\text {nd }}$ ed. Chichester: John Wiley and Sons 1998.

[19] Chapman JW. Learning disabilities in New Zealand: Where kiwis and kids with LD can't fly. J Learn Disabil 1992; 25(6): 362-70.

[20] Lucid Research Ltd. LADS: A new computerised dyslexia screening system for adults. n.d. [cited 2009 May 23]. Available from: http://www.lucid-research.com/documents/factsheets/FS17ن LADSV̈generalV̈description.pdf

[21] Singleton C, Horne J, Simmons F. Computerised screening for dyslexia in adults. J Res Read 2009; 32(1): 137-52.

[22] Jastak S, Wilkinson G. Wide Range Achievement Test. $3^{\text {rd }}$ ed. Los Angeles, CA: Western Psychological Services 1993.

[23] Nichols SA, McLeod JS, Holder L, McLeod HST. Screening for dyslexia, dyspraxia and Meares-Irlen syndrome in higher education. Dyslexia 2009; 15: 42-60.

[24] Miles T. The Bangor Dyslexia Test. Cambridge: Learning Development Aids 1997.

[25] Nicolson RI, Fawcett AJ. Dyslexia Adult Screening Test (DAST). London: Psychological Corporation 1998.

[26] Auckland University of Technology. 2008 Annual report. 2009 [cited 2009 August 23]. Available from: http://www.aut.ac. nz/data/assets/pdfVfile/0010/57547/annual-report-2008.pdf

[27] Disability Resource Service. Learning disabilities. n.d. [cited 2009 May 17]. Available from: Auckland University of Technology website:

http://www.aut.ac.nz/students/studentV̈services/disabilityV services/learning $\dot{V}$ disabilities.htm

[28] Zepke N, Leach L, Brandon J, et al. Standards-based assessment in the senior secondary school: A research synthesis. 2005 [cited 2009 May 23]. Available from: http://www.nzqa.govt.nz/news/features andspeeches/docs/stds-based-assess-litreview.pdf

[29] Human Rights Commission. Human rights in New Zealand today: Nga Tika Tangata O Te Motu. n.d. [cited 2009 May 18]. Available from: http://www.hrc.co.nz/report/chapters/chapter05/disabled02. html

[30] Kaartinen-Koutaniemi M, Katajavuori N. Enhancing the development of pharmacy education by changing pharmacy teaching. Pharm Educ 2006; 6(3): 197-208. 
[31] Reed MJ, Lewis T, Lund-Lucas E. Access to post-secondary education and services for students with learning disabilities: Student, alumni and parent perspectives from two Ontario universities. Higher Educ Perspect 2006; 2(2): 50-65.

[32] University of Wales Institute. Information for students with disabilities. Cardiff, Wales: UWIC 2000.

[33] Fernie T, Henning MA. From a disabling world to a new vision. In: Adams M, Brown S, editors. Towards inclusive learning in higher education: Developing curricula for disabled students. Essex, UK: National Disability Team 2006; pp. 23-32.

[34] Putnam JW, Spiegel AN, Bruininks RH. Future directions in education and inclusion of students with disabilities: A Delphi investigation. Except Chil 1995; 61(6): 553-77.
[35] Ditterline J, Banner D, Oakland T, Becton D. Adaptive behavior profiles of students with disabilities. J Appl School Psychol 2008; 24(2): 191-208.

[36] New Zealand Qualifications Authority. Rules and procedures for tertiary providers assessing against achievement standards 2005 . 2005 [cited 2009 May 23]. Available from: New Zealand Qualifications Authority website: http://www.nzqa.govt.nz/ncea/ acrp/tertiary/docs/rules-procedures-tertprov05.doc

[37] Forgrave KE. Assistive technology: Empowering students with learning disabilities. Clear House 2002; 75(3): 122-45.

[38] The Australian National University. Disability Services Centre. 2009 [updated February 23 cited 2009 May 25]. Available from: http://www.anu.edu.au/disabilities/

(C) Marcus Henning.; Licensee Bentham Open

This is an open access article licensed under the terms of the Creative Commons Attribution Non-Commercial License (http: //creativecommons.org/licenses/by$\mathrm{nc} / 3.0 /$ ) which permits unrestricted, non-commercial use, distribution and reproduction in any medium, provided the work is properly cited. 